CUAD. CONTAB. / BOGOTÁ, COLOMBIA, 15 (38): 575-597 / JULIO-DICIEMBRE 2014 / 575

\title{
Una aproximación al método cartesiano. Su relación con la contabilidad*
}

doi: 10.11144/Javeriana.cc15-38.uamc

\section{Carlos Mario Vargas-Restrepo}

Contador público, Universidad Católica de Oriente. Espe-

cialista en gestión tributaria, Universidad de Antioquia.

Magíster en gestión de organizaciones, Université du

Québec à Chicoutimi, UQAC y Universidad EAN. Estu-

diante del doctorado en ciencias contables, Universidad

de los Andes, Mérida. Docente-investigador, Universidad

Católica de Oriente.

Correo electrónico: cvargas@uco.edu.co;

cvargas12@gmail.com

\footnotetext{
* Artículo resultado de investigación. El artículo se deriva del macroproyecto de investigación Proyecto ECO - Difusión del conocimiento y transferencia de tecnología para el desarrollo regional, que tiene como objetivo articular las diferentes acciones académicas y medios disponibles de la Facultad de Ciencias Económicas y Administrativas de la Universidad Católica de Oriente, encaminadas a la difusión del conocimiento y transferencia de tecnología, para consolidar espacios que contribuyan al aprendizaje social y desarrollo regional. En este macroproyecto se realizó una investigación orientada a analizar los planteamientos cartesianos y la influencia de estos en el desarrollo de la contabilidad. De ahí surge la escritura del artículo. La investigación tuvo una duración de seis meses y estaba concluida al momento de enviar el artículo a la revista. Este proyecto está inscrito ante la Dirección de Investigación y Desarrollo de la Universidad Católica de Oriente (institución financiadora).
} 


\section{Resumen La filosofía cartesiana constituyó el punto} de partida de la modernidad. Con ella, se impone la razón en la manera de aproximarse al conocimiento y cobra relevancia lo medible y lo cuantificable, legado de los postulados de Galileo Galilei. Así entonces, los preceptos del método cartesiano y la duda metódica sirvieron de base para acceder al conocimiento verdadero y para descubrir nuevas verdades a partir de las ya conocidas. El desarrollo de la disciplina contable también estuvo influenciado por la filosofía de René Descartes fundamentalmente en lo que respecta a la racionalidad que consolidó la tradición investigativa en contabilidad (investigación positivista) y a las diferentes prácticas contables que se basan en la lógica de lo medible y lo cuantificable. El análisis se desarrolla sobre estas bases con el propósito de efectuar una aproximación a los postulados de Descartes y la relación que pudiera existir entre estos y la disciplina y la práctica contables.

Palabras clave Método cartesiano; duda metódica; filosofía cartesiana; contabilidad; disciplina contable

\section{Código JEL M 40}

\section{An Approach to the Cartesian Method Its Relation with Accounting}

Abstract The Cartesian philosophy established the starting point of the modern era; with it, reason takes precedence as the approach to knowledge and the measurable and the quantifiable became relevant, a legacy of the postulates of Galileo Galilei. So, in this way, the rules of the Cartesian method and the Cartesian doubt served as the basis to gain access to the true knowledge and to discover new truths from those already known. The development of the accounting discipline was also influenced by the philosophy of Rene Descartes, basically regarding to rationality, which consolidated the research tradition on accounting (positivist research), and the different accounting practices, based on the logic of what can be measured and quantified. Based on the foregoing, I perform the analysis with the purpose of making an approach to the postulates of Descartes and the possible relationship that might exist between them and the accounting discipline and practice.

Keywords Cartesian method; Cartesian doubt; Cartesian philosophy; accounting; accounting discipline

\section{Aproximação ao método cartesiano. Sua relação com a contabilidade}

Resumo A filosofia cartesiana constituiu o ponto de partida da modernidade. Com ela, impõe-se a razão na maneira de se aproximar do conhecimento e cobra relevância o mensurável e o quantificável, legado dos postulados de Galileu Galilei. Assim, então, os preceitos do método cartesiano e a dúvida metódica serviram de base para aceder ao conhecimento verdadeiro e para descobrir novas verdades a partir das já conhecidas. O desenvolvimento da disciplina contábil também esteve influenciado pela filosofia de René Descartes fundamentalmente no que diz respeito da racionalidade que consolidou a tradição pesquisadora na contabilidade (pesquisa positivista) e as diferentes práticas contábeis baseadas na lógica do medível e o quantificável. A análise se desenvolve sobre estas bases com o propósito de efetuar uma aproximação aos postulados de Descartes e a relação que pudesse existir entre estes e a disciplina e prática contábeis.

Palavras-chave Método cartesiano; dúvida metódica; filosofia cartesiana; contabilidade; disciplina contábil

\section{Introducción}

El presente trabajo pretende exponer los principales planteamientos de la filosofía cartesiana y su relación con la disciplina contable. A partir de René Descartes, se dio inicio a una filosofía estrictamente moderna, la cual se ha caracterizado por un alto grado de racionalismo. 
Con todo, el pensamiento cartesiano significó rupturas considerables en la base filosófica que se había construido a lo largo de la historia y que se había consolidado durante la época medieval. En efecto, rompe con el paradigma heliocéntrico en la forma de construir el conocimiento y pone de relieve la razón, incluso por encima del sujeto mismo. No interesa este, sino lo que conoce por medio de la razón.

La propuesta de René Descartes sintetizada en el método para hacer ciencia (método cartesiano), tuvo influencia en todas las áreas del conocimiento. Puede señalarse que en contabilidad también se apropiaron elementos de la filosofía cartesiana, sobre todo por parte de la corriente que soporta la tradición investigativa contable (investigación positivista). El método de Descartes también se caracterizó por adoptar el modelo matemático (deducción) con el que se confirmó de alguna manera lo expuesto por Galileo Galilei, en el sentido de que la naturaleza estaba escrita en el lenguaje de las matemáticas. De ahí que muchas prácticas contables se sustenten en la medición y cuantificación de los hechos para su reconocimiento, revelación y análisis como se explica posteriormente en este documento.

Así, además de pretender efectuar una aproximación al método cartesiano, se quiere identificar la posible relación entre este y la disciplina contable, sobre todo en lo que concierne a la racionalidad que contribuyó a la consolidación de la investigación contable predominante (ortodoxa) y de las diferentes prácticas contables que se basan en la lógica de lo medible y lo cuantificable. Aunque las exposiciones incluidas en el artículo se hacen sobre estas bases, se reconoce la existencia de otros enfoques incor- porados en la contabilidad (heterodoxos) como el interpretativo o el sociocrítico, que, aunque no se explican ampliamente en este escrito por limitaciones de espacio, es importante señalar que han permitido superar el paradigma positivista de la ciencia y producir conocimiento desde otras perspectivas en las que la subjetividad también es importante para conseguirlo.

En este sentido, la subjetividad hace referencia a la separación de los enfoques cuantitativosobjetivistas prevalecientes en la investigación contable, para dar cabida a perspectivas en las que la aproximación a la realidad se efectúa bajo otras concepciones, como que esta (la realidad) es socialmente construida o que corresponde a una proyección de la imaginación humana. De una parte, la construcción social de la realidad refiere a enfoques subjetivistas en los que "son las acciones sociales las que centran la investigación y los investigadores se ocupan de los procedimientos a través de los cuales los actores individuales comprenden "qué está pasando"' (Ryan, Scapens \& Theobald, 2004, p. 55), lo que genera la posibilidad de varias realidades. Por otro lado, la realidad como una proyección de la imaginación humana corresponde a la "posición subjetivista extrema en la que la realidad existe solo en la conciencia individual, o sea, en la imaginación humana” (Ryan, Scapens \& Theobald, 2004, p. 55).

Ahora bien, los argumentos y discusiones expuestas en el documento se plantean a partir de la metodología hermenéutica concebida como "la disciplina de la interpretación. La interpretación es la comprensión, vista aquí [...], como un proceso, no como un acto instantáneo y definitivo, sino que va profundizando sucesivamente en lo que interpreta" (Beuchot, 2008, p. 33). 
Desde la hermenéutica, se apeló al criterio o método del análisis del discurso con el cual se pretendió interpretar algunos textos - obras de Descartes- para comprender las tesis del autor y abstraer la relación que pudiera existir entre sus planteamientos y la disciplina contable, como se indicó anteriormente. La argumentación no se extiende a todas las áreas de desarrollo de la contabilidad, pues se está consciente de la emergencia de nuevos paradigmas que, como ya se anotó, desbordaron la lógica matemática y racional de la filosofía cartesiana.

Desde esta perspectiva, el escrito está estructurado en cinco partes. En la primera de ellas se hace una contextualización de la filosofía cartesiana; en la segunda, se expone el método cartesiano y en la tercera, la duda metódica. En el cuarto acápite se hace una aproximación a la relación existente entre el método cartesiano y la racionalidad propuesta por Descartes, y la contabilidad y la influencia que esta ha recibido de ese pensamiento filosófico. Por último, se esboza la existencia de perspectivas heterodoxas que se separan del racionalismo para dar cuenta de manera diferente - no menos importante-de los fenómenos de la realidad.

El trabajo busca, en últimas, suscitar la reflexión en torno a la contabilidad, a fin de contribuir a la consolidación de la investigación contable y de la comunidad académica en el país.

\section{Contextualización y aspectos preliminares}

La ciencia moderna tiene sin duda como su referente más remoto la física aristotélica. En efecto, la filosofía de este griego ha permea- do los trabajos de Nicolás Copérnico, Isaac Newton, Galileo Galilei, René Descartes y de los teóricos del positivismo actual y del empirismo lógico (Carvajal, 2007, p. 17). Sin embargo, en algunos otros momentos y de manera antagónica, los postulados platónicos han influenciado el pensamiento de otros filósofos, incluso Descartes parece introducir en sus teorías algunos de los planteamientos sobre las ideas del griego Platón.

En este contexto, el pensamiento cartesiano surge como una respuesta al momento de incertidumbre suscitado entre los siglos XV y XVII, cuando pierden vigencia el modelo científico geocentrista (que predicaba a la tierra como centro del universo) y el teocentrista (Dios como centro de la realidad y de todo pensamiento humano), y nace un nuevo modelo de ver el universo, y un nuevo método de pensar sustentado en proposiciones matemáticas: el heliocentrismo, según el cual cobra relevancia y se impone lo que se puede medir y cuantificar.

Además de esto, en esa época se generan fragmentaciones en el catolicismo, que dieron origen a la "duda" y esta a su vez puso en tela de juicio el criterio de verdad y las estructuras que se consideraban su fuente: la religión $(f e)$ y la ciencia (razón). De esta manera, esta época de la historia estuvo marcada por el escepticismo. Frente a esto, Édgar Perilla-Amórtegui y Marcos Valderrama-Prieto (1996) exponen lo siguiente:

Con el advenimiento del Renacimiento se da un cambio en el retorno al estudio de la filosofía clásica, en la medida en que se producen los descubrimientos intercontinentales, el avance de la física y las matemáticas; de- 


\section{UNA APROXIMACIÓN AL MÉTODO CARTESIANO. SU RELACIÓN CON LA CONTABILIDAD / C. VARGAS / $\mathbf{5} 79$}

jan de tener validez dogmas hasta entonces considerados eternos, como el geocentrismo, y nace en muchos la duda sobre lo que es realmente verdadero (p. 19).

Para contrarrestar este manto de incertidumbre y duda, Descartes pretendió establecer una estructura filosófica en la que no hubiese ningún tipo de error. Las matemáticas le ayudaron en este propósito — de ahí su pasión por esta área de la ciencia-, pero además encontró en la razón humana el piso firme sobre el cual plantar su pensamiento y la nueva estructura filosófica que propuso. Los planteamientos filosóficos de Descartes aparecen en un contexto marcado por el derrumbamiento de la escolástica como sistema de ideas y creencias que había imperado durante varios siglos.

Así, se considera a René Descartes como el padre de la filosofía moderna y como el precursor del racionalismo científico, pues según él, solo la razón es capaz de diferenciar lo verdadero de lo falso, mientras los sentidos generan engaño y conducen al error (Hernández \& Salgado, 2010-2011).

Igualmente, Descartes pretendió generar una unificación de todas las ciencias, no en su objeto de estudio sino en el método, pues consideraba que el error se presentaba no por la falta de inteligencia de los seres humanos (investigadores y científicos), sino por los pasos que se dan para descubrir la verdad. El mejor método, según el filósofo, era el brindado por las matemáticas "por la certeza y evidencia que poseen sus razones" (Descartes, 2001, p. 43). Desde esta perspectiva, se entiende su famosa metáfora cartesiana, en la que las diferentes ciencias forman una unidad al igual que un árbol, y por ello en este pensador el saber tiene un carácter orgánico, es decir, las diferentes expresiones de conocimiento se relacionan entre sí:

Toda filosofía es como un árbol cuyas raíces son la Metafísica, el tronco es la física y las ramas que salen de ese tronco son todas las otras ciencias, que se reducen a tres principales, a saber, la Medicina, la Mecánica y la Moral (Monroy-Nasr, citando a Descartes, 2004, p. 3).

El legado de Descartes, plasmado en sus diferentes obras, devela el carácter científicoracional de este filósofo y el escepticismo como uno de sus rasgos personales. Sin embargo, tal escepticismo tiene qué ver con la necesidad de conocimientos verdaderos y con dudar de todo mientras no se tenga evidencia de que sea certero.

En este sentido, el escepticismo de Descartes no hace referencia a los planteamientos de la escuela conocida con este mismo nombre (los escépticos), según la cual no hay posibilidad de acceder a la verdad. En efecto, Johannes Hessen (1997) señala que para la escuela filosófica que defiende el escepticismo, "el sujeto no puede aprehender el objeto. El conocimiento, en el sentido de una aprehensión real del objeto, es imposible según él. Por eso no debemos pronunciar ningún juicio, sino abstenernos totalmente de juzgar" (p. 41).

En René Descartes, a contrario sensu, es posible acceder al conocimiento y reconoce que a este se puede arribar por dos vías: por la expe- 
riencia (concebida también como intuición) y por la deducción. Lo anterior no significa que le esté dando relevancia al conocimiento sensible ${ }^{1}$ y quizás por ello en el desarrollo de sus tratados reemplaza la experiencia por la intuición, pues esta junto con la deducción, son cualidades de la razón y en esta medida pueden proporcionar conocimientos ciertos. De hecho, la experiencia - advierte el filósofo-puede ser engañosa puesto que los sentidos pueden hacer percibir y comprender la realidad de manera equivocada. De ahí su apego por las matemáticas, ciencia que procede desde la óptica deductiva y elabora el saber a partir de la mente sin tomar nada de la experiencia (Descartes, 1984).

Ahora bien, Descartes (1984) define su concepción de intuición en los siguientes términos:

Entiendo por intuición, no el testimonio fluctuante de los sentidos, o el juicio falaz de una imaginación que compone mal, sino la concepción de una mente pura y atenta tan fácil y distinta, que en absoluto quede duda alguna sobre aquello que entendemos; o, lo que es lo mismo, la concepción no dudosa de una mente pura y atenta, que nace de la sola luz de la razón (p. 75).

Por otra parte, Descartes (1984) entiende la deducción como "todo aquello que se sigue necesariamente de otras cosas conocidas con certeza" (p. 76).

1 El conocimiento sensible es promulgado por la escuela del empirismo, según la cual la única fuente del conocimiento humano es la experiencia. Es la antítesis de los planteamientos de la escuela del racionalismo, para la cual la razón y el pensamiento constituyen la fuente principal del conocimiento.
De otro lado, el racionalismo formulado por Descartes tiene dos aspectos característicos y diferenciadores. En primer lugar, está lo epistemológico-metodológico, en el entendido de que la mayor preocupación cartesiana estaba en buscar un nuevo método de pensar y construir el pensamiento filosófico acogiendo el modelo matemático. Esto corresponde a la "duda metódica" planteada por Descartes.

En segundo lugar, lo metafísico también estaba presente en el pensamiento cartesiano al centrarse en conceptos como los de verdad y sustancia. Era necesario descubrir las verdades fundamentales, y así Descartes descubrió la primera de ellas: el cogito. Igualmente, esta metafísica conduce a un nuevo paradigma sobre el funcionamiento del universo (mecanicismo) y a una nueva teoría de las ideas.

En la figura 1 se puede observar la concepción que tenía Descartes de la ciencia y, de alguna manera, su pensamiento filosófico. De acuerdo con esta figura, la ciencia se construye a partir del método cartesiano, el cual consiste en los siguientes pasos: el primero de ellos sustentado en la evidencia, a partir del cual solo pueden aceptarse conocimientos certeros y rechazar aquellos que son solo probables. Por tanto, ha de dudarse de casi todo —duda metódica-a menos que sea para el ser humano claro y distinto. Respecto de esto último, el mismo Descartes expuso:

llamo claro a aquel conocimiento que está presente y manifiesto a un espíritu atento [...] y distinto a aquel que siendo claro, es de tal manera preciso y diferente de todos los demás, que no comprende en sí sino aquello que manifiestamente aparece a aquel que le 


\section{UNA APROXIMACIÓN AL MÉTODO CARTESIANO. SU RELACIÓN CON LA CONTABILIDAD / C. VARGAS / 581}

considera como conviene (Hirschberger, citando a Descartes, 2000, p. 35).

De esta manera, la claridad es diferente a la distinción pues "conocer con distinción, supone estar en condiciones de mostrar todos y cada uno de los atributos que pertenecen a un sujeto" (Quintás-Alonso, Gracia-Calandín, Larraz, Moreno, Palanca \& Ruiz, 2009, p. 60). De acuerdo con los mismos autores, "esto es lo que autoriza a Descartes a afirmar que quien conoce un triángulo como una figura formada por tres lados, pero, por ejemplo, no conoce el valor de sus ángulos, no posee un conocimiento claro y distinto" (Quintás-Alonso, Gracia-Calandín, Larraz, Moreno, Palanca \& Ruiz, 2009, p. 60).

Posteriormente, se deben dejar funcionar las ya mencionadas cualidades de la razón: la intuición y la deducción, para dar cabida a los pasos dos y tres del método que consisten en el análisis y la síntesis respectivamente.

Por último, en el cuarto paso, se hacen revisiones de los pasos previos y de las enumeraciones a fin de recordar todo el proceso de construcción del conocimiento. Este método y otros postulados relacionados con el racionalismo cartesiano son desarrollados con mayor detalle en los siguientes apartes.

\section{La duda metódica}

El método propuesto por Descartes - a exponer en el siguiente aparte- se basa en la duda, de tal manera que se debe considerar falso todo aquello en lo que se encuentre la más mínima probabilidad de incertidumbre. La duda encuentra su origen en el mismo ser humano. El sujeto es el fundamento de todo saber, pues la principal fuente del conocimiento está en la razón.

Como se explicó anteriormente, la duda en el discurso cartesiano es diferente al escepticismo. Por ello, en Descartes se configura la denominada "duda metódica", a partir de la cual solo se debe dar cabida a lo verdadero, es decir, al conocimiento adquirido de manera clara, distinta, evidente y certera. El conocimiento probable se debe rechazar, pues ha de considerarse falso. Así entonces, la duda metódica constituye el aspecto epistemológico del método cartesiano en cuanto esta se refiere a la pregunta por la verdad. Por otra parte, el método en sí corresponde al aspecto metodológico de la filosofía cartesiana, ello es, los pasos para acceder al conocimiento verdadero.

La duda en este contexto reúne las características de universal y metódica. En el primer caso, se hace referencia a la necesidad de dudar de casi todo (de los sentidos, del mundo exterior, de los propios razonamientos, de sí mismo, etc.), mientras en el segundo caso se quiere diferenciar del escepticismo. En Descartes, sí es posible acceder al conocimiento verdadero y la duda es tan solo un mecanismo para conseguirlo.

Descartes dedica parte de su discurso del método y su primera Meditación metafísica a plantear la necesidad de dudar y señala que si bien los sentidos sirven de instrumento para adquirir el conocimiento, en ocasiones pueden engañar la mente y producir por tanto verdades falsas. De ahí, la total relevancia que da a la razón como única posibilidad de construir conocimientos verdaderos, pues esta halla la verdad por medio del método descrito anteriormente, es decir, correspondería a un saber racional. 


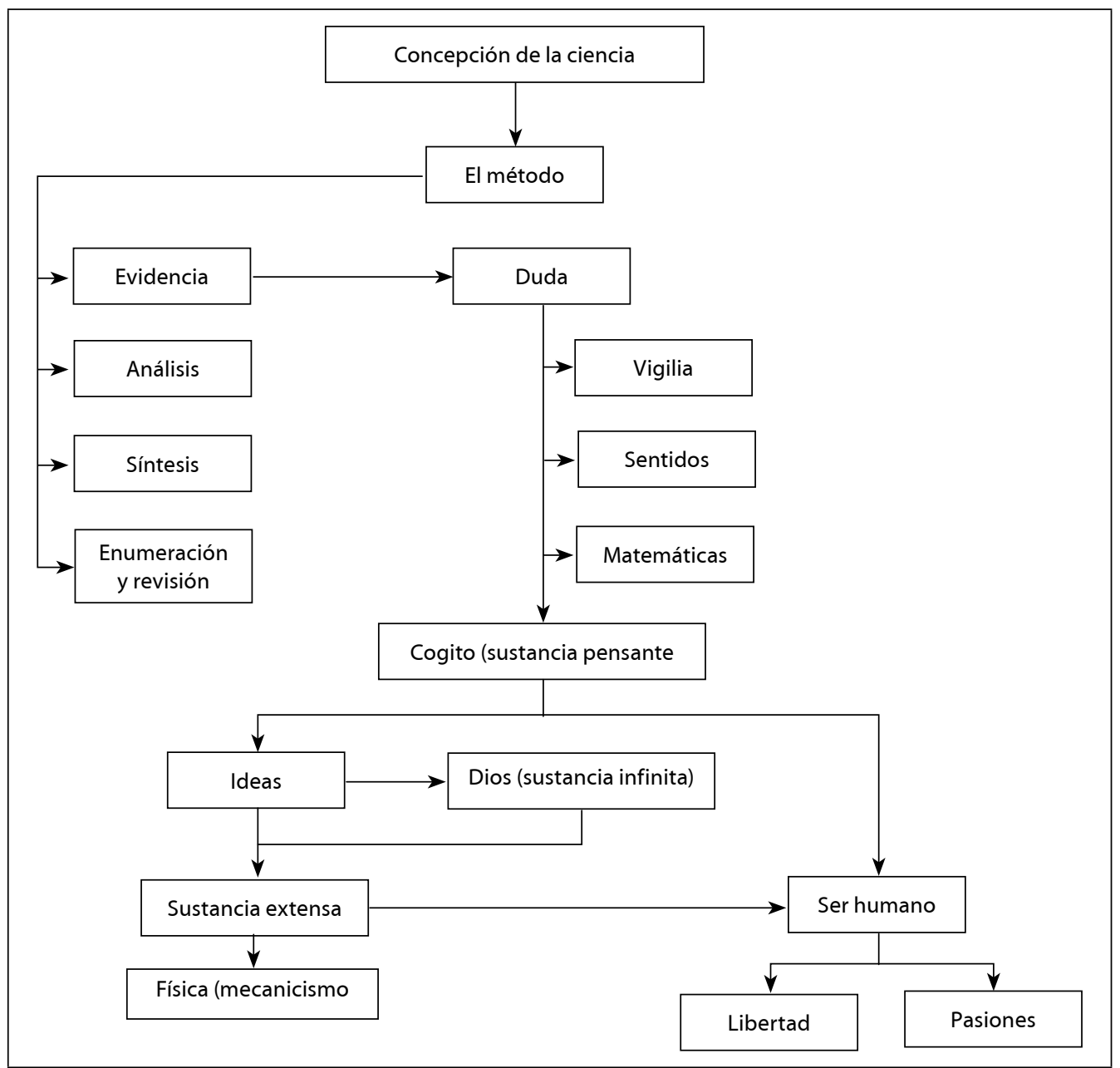

Figura 1. Concepción de la ciencia por Descartes Fuente: Manuel Luna-Alcoba (2003)

En su proceso de dudar de todo, René Descartes descubre la primera verdad esencial: el hombre es un ser pensante (cognoscente). No se puede dudar que el hombre duda, es decir, que piensa. Aún más, se puede dudar de lo pensado mas no del pensamiento mismo. De esta manera, llega a esta primera verdad: "cogito, ergo sum" que significa "pienso, luego existo", proposición esta que configura la base prime- ra (inicio) de toda la filosofía de Descartes. Por ello, se puede señalar que esta proposición acentúa la propuesta de cambio del esquema de la filosofía, al pasar del objetivismo de la época medieval al subjetivismo planteado en el método cartesiano, en virtud del cual lo importante es el conocimiento de las cosas por medio de la razón humana. El pensamiento se convierte en el punto de partida del conoci- 
miento, ahora elaborado de manera racional. Esta primera verdad nace de la intuición y está sustentada en la certeza, toda vez que es clara y evidente.

Con la duda metódica, el sujeto pensante (cogito) pasa a ser la base sobre la que se construye todo el saber racional; y con la primera verdad, se descubre también la existencia del hombre. Si yo pienso, es porque existo; existo como un ser pensante. Para dudar hay que pensar, y para pensar hay que existir (Descartes, 2001).

\section{El método cartesiano}

Entre las obras de Descartes, sobresalen dos que dan cuenta con más énfasis de la necesidad de acceder a la verdad de manera ordenada, es decir, con un método que oriente el camino a recorrer para obtener como propósito central un conocimiento sustentado en la verdad, la evidencia y la certeza; pero además que elimine cualquier posibilidad de confusión, incertidumbre y error. Se hace referencia a las obras: Discurso del método para dirigir bien la razón y buscar la verdad en las ciencias (Descartes, 2001) y Reglas para la dirección del espíritu (Descartes, 1984), a partir de las cuales se pretende fundamentar este escrito.

En primera instancia, es importante resaltar que para Descartes la ciencia y la filosofía guardan una estrecha relación, lo cual hace evidente no solo por su conocida metáfora del árbol — citada anteriormente-, sino también por su consideración de que todas las ciencias y disciplinas se construyen a partir del conocimiento que desarrolla el hombre y tienen un núcleo en común: el método.
De otro lado, el método garantiza la consecución acertada de la verdad, porque proporciona seguridad en el proceso de su obtención y aminora el riesgo de caer en el error. No se puede admitir el hecho de que se haga o se construya ciencia sin un método, este "es necesario para la investigación de la verdad de las cosas" (Descartes, 1984, p. 78). Los científicos, investigadores y filósofos que demuestran verdades en ausencia de este, las deben más a cuestiones del azar y son, por tanto, más vulnerables a los errores, lo que finalmente repercute en el grado de confiabilidad de tales verdades y en el grado de rigurosidad del trabajo científico.

El método, por tanto, se puede considerar como la posibilidad que tiene el sujeto de acercarse al objeto. Descartes (1984) plantea que en el conocimiento de las cosas intervienen dos actores: el ser humano que conoce y las cosas que deben ser conocidas. El hombre conoce por el entendimiento, la imaginación, los sentidos y la memoria, tesis esta que también estuvo presente en la filosofía platónica y aristotélica. Del objeto hay que examinar lo que se muestra como tal y lo que se deduce a partir de lo conocido $^{2}$. Para que el sujeto cognoscente descubra las verdades del objeto requiere del método. Así entonces, Descartes (1984) enseña que el método consiste en un conjunto de:

\footnotetext{
2 Johannes Hessen (1997) plantea que la filosofía corresponde a un movimiento pendular entre la concepción del yo (sujeto) y la concepción del universo (objeto). "Pero ello prueba precisamente que ambos elementos pertenecen a aquel concepto esencial. No se trata de una alternativa (o el uno, o el otro), sino de una acumulativa (tanto el uno como el otro). La filosofía es ambas cosas: una concepción del yo y una concepción del universo" (1997, p. 18).
} 
reglas ciertas y fáciles, mediante las cuales el que las observe exactamente no tomará nunca nada falso por verdadero, $y$, no empleando inútilmente ningún esfuerzo de la mente, sino aumentando siempre gradualmente su ciencia, llegará al conocimiento verdadero de todo aquello de que es capaz (p. 79).

En la figura 2 se pueden observar los pasos del método cartesiano.

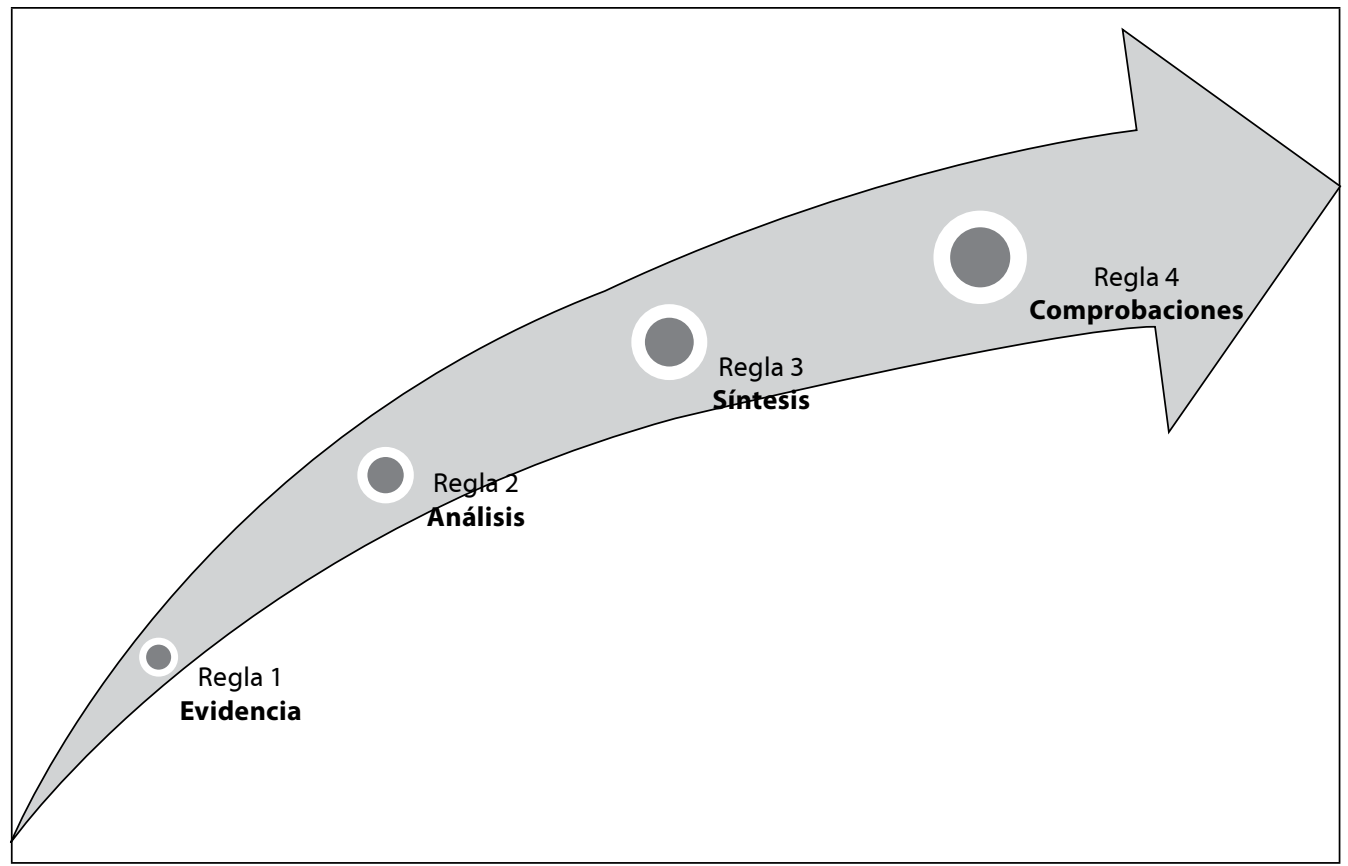

Figura 2. Reglas del método cartesiano

Fuente: elaboración propia con base en René Descartes (2001)

Por considerarlo de relevancia, a continuación se presentan cada una de las reglas del método escritas por Descartes (2001) en el Discurso del método:

1. Regla de la evidencia: "No admitir como verdadera cosa alguna, como no supiese con evidencia que lo es; es decir, evitar cuidadosamente la precipitación y la prevención, y no comprender en mis juicios nada más que lo que se presentase tan clara y distintamente a mi espíritu, que no hubie- se ninguna ocasión de ponerlo en duda" (p. 52).

2. Regla del análisis: "Dividir cada una de las dificultades que examinase en cuantas partes fuera posible y en cuantas requiriese su mejor solución” (p. 52).

3. Regla de la síntesis: "Conducir ordenadamente mis pensamientos, comenzando por los objetos más simples y más fáciles de conocer, para ir ascendiendo poco a poco, gradualmente hasta el conocimiento de los 


\section{UNA APROXIMACIÓN AL MÉTODO CARTESIANO. SU RELACIÓN CON LA CONTABILIDAD / C. VARGAS / 585}

más compuestos, e incluso suponiendo un orden entre los que no se preceden naturalmente" (p. 53)

4. Regla de las comprobaciones: "Hacer en todos unos recuentos tan integrales y unas revisiones tan generales, que llegase a estar seguro de no admitir nada" (p. 53).

Esta última regla sería algo así como efectuar las enumeraciones y revisiones necesarias respecto al objeto analizado para asegurarse de que es realmente verdadero.

La propuesta descrita en el método cartesiano pretende de una parte evitar el error y obtener verdades (intuición), es decir, discernir lo verdadero de lo falso; y de otra, hallar nuevas verdades a partir de las ya existentes y conocidas (deducción). Esto es imperativo para Descartes y lo consagra tanto en la primera $\mathrm{Me}$ ditación metafísica - de las cosas que se pueden poner en duda - como en la primera parte del Discurso del método y en la Segunda regla para la dirección del espíritu ${ }^{3}$, insistiendo reiterativamente en la necesidad de rechazar el error y de aceptar solo la verdad. En efecto, Descartes (1984) señaló en la citada Segunda regla que "rechazamos todos aquellos conocimientos tan solo probables y establecemos que no se debe

3 En la primera Meditación metafísica, Descartes (2001) señala: "la razón me persuade ya de que debo ocuparme no menos cuidadosamente en dar crédito a las cosas que no son enteramente ciertas e indudables que a las que me parezcan ser manifiestamente falsas, esto me bastará para rechazarlas todas si puedo encontrar alguna razón para dudar en cada una" (p. 124).

Por su parte, en la primera parte del Discurso del método, escribió que "sentía un deseo extremado de aprender a distinguir lo verdadero de lo falso, para ver claro en mis actos y andar seguro por esta vida" (p. 45). dar asentimiento sino a los perfectamente conocidos y de los que no puede dudarse" (p. 67).

En este sentido, la intuición y la deducción se entienden como facultades de la razón humana y en esta medida, se deben dejar actuar en el método a fin de llegar a la verdad. Ambas facultades están presentes en los pasos del método cartesiano; la intuición en los dos primeros y la deducción en los dos últimos. En Descartes, ambas facultades cobran relevancia porque son el único modo de acceder al conocimiento y por ello sustentan este método. En la Novena regla, el filósofo deja descubrir dos facultades del espíritu relacionadas estrechamente con las facultades en cuestión: "la perspicacia, intuyendo distintamente cada cosa, y la sagacidad, deduciendo con arte unas de otras" (Descartes, 1984, p. 107).

Se puede inferir entonces que la intuición en el método cartesiano corresponde a la parte analítica y la deducción a la parte sintética. Por tanto, en términos generales, podría anotarse que el método no es más que la aplicación de dos fórmulas a una pregunta de investigación (objeto de estudio o fenómeno natural) para descubrir verdades. Estas fórmulas son el análisis y la síntesis.

Ahora bien, para poner en acción la intuición, se exigen dos condiciones. La primera hace referencia a que lo intuido debe ser entendido clara y distintamente; y la segunda, que lo que se intuye se haga al mismo tiempo y no de manera sucesiva. La deducción, por el contrario, se hace en un movimiento de la mente a partir del cual se infiere una cosa de otra, por tanto no es simultáneo sino progresivo. No obstante, en la aplicación del método llega un 
punto en el que el sujeto después de aproximarse a la verdad, logra fusionar su facultad deductiva con la inductiva, de tal suerte que recorre los pasos del método de manera rápida distinguiendo lo verdadero de lo falso y dando la impresión de que esto último lo hace solo desde la perspectiva inductivista (Descartes, 2001).

En el marco de estos planteamientos, se comprende la necesidad que sintió Descartes de eliminar todo conocimiento anterior y construir uno nuevo cuyo piso fuera solo la verdad. Haciendo un símil con una de las actuales teorías en educación y pedagogía, es desaprender para aprender de nuevo sin influencia de prejuicios y sin verdades que no contengan fundamento, las cuales pueden contener algún tipo de error. Johannes Hessen (1997) tiene un planteamiento que ayuda a dar claridad al concepto de verdad, según el cual esta "se relaciona estrechamente con la esencia del conocimiento [...]. Un conocimiento falso no es propiamente conocimiento, sino un error e ilusión" (p. 32). Más adelante, señala el mismo autor que "la verdad es la concordancia del pensamiento consigo mismo [...]. La verdad del conocimiento solo puede consistir, por ende, en la producción correcta - conforme a las leyes-del objeto, esto es, en que el pensamiento concuerde con sus propias leyes" (pp. 122-123), planteamientos estos muy en favor de la visión cartesiana como se expondrá posteriormente.

Sin embargo, respecto del problema de la verdad en el contexto del conocimiento, de manera contraria a los postulados de Hessen (y hasta del mismo Descartes), deben considerarse otras posturas, una de ellas, la de Gaston Bachelard (referenciado por Ávila, 2005) para quien "las verdades producidas por la ciencia, se establecen a lo largo de un proceso" (p. 132). Por ello, afirma Francisco Ávila-Fuenmayor (2005) lo siguiente:

la filosofía idealista manifiesta constantemente que 'busca' la verdad; y si no la busca la cuestiona, la desvela. En cualquier caso, existe una estrecha relación con ella. Sin embargo, se llega a comprender su verdad cuando se haya descubierto en la pareja Sujeto-Objeto. Así, se dice que un conocimiento es verdadero, es decir, científico, cuando Sujeto $=$ Objeto Esta doble reflexión de una doble presencia es el conocimiento. En esta misma línea de pensamiento, postula, que una verdad no puede ser considerada en forma absoluta en cuanto a ser considerada como el punto de perfección del conocimiento sino que siempre es un tramo más de un proceso permanente de aproximaciones (p. 133).

El planteamiento anterior constituye uno de los cuestionamientos elaborados en torno al racionalismo que se impuso durante bastante tiempo en la ciencia a partir del pensamiento de Descartes. Posteriormente, se retomará para hacer notar la importancia de perspectivas alternativas a la racionalidad científica.

Por ahora, es necesario develar el criterio o concepto de Descartes respecto a la verdad. Puede afirmarse que en la filosofía cartesiana, la verdad está estrechamente relacionada con la certeza. En efecto, el primer precepto del método concierne a la evidencia y esta, dado que no permite aceptar el conocimiento tan solo 
probable o del que se tenga incertidumbre, asegura la certeza en la obtención de la verdad. Se elimina la duda, el error, la incertidumbre y se acepta solo lo que es ciertamente verdadero. El criterio de certeza, por su parte, está identificado — como se mencionó anteriormente-con la claridad y distinción en los conceptos, esto es, en el conocimiento. De hecho, Descartes (1984) reitera en la Tercera regla para la dirección del espíritu que "acerca de los objetos propuestos se ha de buscar no lo que otros hayan pensado o lo que nosotros mismos conjeturemos, sino lo que podamos intuir clara y evidentemente o deducir con certeza; pues la ciencia no se adquiere de otra manera" (1984, p. 72).

En este punto de la exposición, cabe recordar que el método cartesiano está fundamentado en las matemáticas, por cuanto es la ciencia que según Descartes (2001) brinda certeza y evidencia en sus razones. El modelo matemático está caracterizado por demostrar verdades a partir de otras (deducción). En efecto, en su Discurso del método, Descartes (2001) llega a afirmar que "entre todos los que hasta ahora han investigado la verdad en las ciencias, solo los matemáticos han podido encontrar algunas demostraciones, esto es, algunas razones ciertas y evidentes" (p. 53). Estos planteamientos han derivado la denominación de matematización del método cartesiano, es decir, la incorporación del modelo matemático al método para acercarse a la verdad planteado por Descartes.

Ahondando un poco más en el análisis del método cartesiano, podría señalarse a la luz del primer paso, el de la evidencia, que esta constituye el criterio con el cual ha de separarse lo verdadero de lo falso. La verdad debe ser evi- dente. El mismo Descartes (2001) señala en su Discurso del método de manera complementaria que "no debemos dejarnos persuadir nunca sino por la evidencia de la razón. Y nótese bien que digo de la razón, no de la imaginación ni de los sentidos" (p. 70). Por consiguiente, puede afirmarse que todo el método se reduce a la evidencia.

Por su parte, los pasos dos y tres (análisis y síntesis) permiten deducir nuevas verdades tomando como punto de partida las ideas obtenidas de manera clara y distinta mediante el paso uno. Así, el segundo paso consiste en descomponer el objeto analizado en sus partes más simples (intuición), acto este por medio del cual la mente humana proporciona conocimientos evidentes (claros y distintos).

Una vez conocido el problema en todas sus partes y la manera como se relacionan entre sí, se aplica el paso tres, en virtud del cual se recompone el objeto de estudio a partir de lo simple. De esta manera, se está en presencia del enfoque deductivo que podría decirse, consiste en una cadena de intuiciones. Desde esta perspectiva, mediante la intuición - primer acto de la razón- se elaboran los conceptos simples mientras que mediante la deducción, se construyen los conceptos relativos y debidamente estructurados. Por ello como se señaló anteriormente, la deducción requiere necesariamente de un proceso progresivo, en razón del cual una cosa (verdad) antecede a otra.

El último paso consiste en una medida de precaución para no olvidar lo realizado en el proceso de descubrir verdades. De acuerdo con este paso, se requiere realizar enumeraciones y revisiones de las etapas seguidas, sobre todo 
en los procesos de análisis y síntesis. En efecto, las enumeraciones verifican de alguna manera el análisis mientras las revisiones comprueban que la cadena de intuiciones esté completa. Varias de las Reglas para la dirección del espíritu insisten en la necesidad de dar un orden ${ }^{4}$ a las cosas y de disponerlas de forma tal que aseguren que serán recordadas (memoria) en cualquier momento y que, aunque se consideren simples, hacen parte del todo analizado y ayudan por tanto a comprenderlo (Descartes, 1984).

De ahí que Descartes da total relevancia a lo fácil e insignificante, toda vez que sirve al pensamiento a acostumbrarse a intuir distinta y claramente la verdad. De hecho, plantea una crítica a quienes tratan las cosas complejas y difíciles con mayor fascinación y asombro, advirtiendo además que "cada uno se persuada firmemente de que deben deducirse las ciencias, aún las más ocultas, no de cosas grandes y oscuras, sino solo de las más fáciles y obvias" (Descartes, 1984, p. 108) $)^{5}$. Sin embargo, es preciso

4 Otros filósofos propusieron un sistema de categorías que permitiese hacer agrupaciones y dar orden al proceso de construcción del conocimiento. Aristóteles, por ejemplo, distinguió diez clases de categorías: sustancia o esencia, cantidad, cualidad, relación, lugar, tiempo, posición, estado, acción y pasión. En Immanuel Kant, hay tantas categorías como clases de juicios puedan distinguirse (Hessen, 1997, p. 142) y construye su sistema a partir de cuatro categorías: cantidad, cualidad, relación y modalidad. Eduard von Hartmann, por su parte, contempla las categorías de la sensibilidad (sensación e intuición) y categorías del pensamiento (reflexivo y especulativo) (Hessen, 1997).

5 Las pasiones del alma es otra obra de Descartes (1997) que da cuenta de su pensamiento en torno a la admiración (artículos 75 a 76). "Se puede decir de la admiración en particular que es útil porque hace que aprendamos y retengamos en nuestra memoria las cosas que hemos ignorado anteriormente" (p. 147); por tanto, la considera como ori- aclarar que no es que se deban dejar de lado las cosas grandes y oscuras, sino más bien que se debe considerar todo, aun aquello que parezca ser simple y obvio. De hecho, en la Décima regla prescribe el filósofo que sin descuidar las cosas sencillas, también hay que enfocarse en las complejas, pues todas integran el objeto de estudio y ambas ayudan a construir ciencia, pero es necesario ir en un proceso desde lo simple pasando por lo más elaborado hasta llegar a la verdad.

De otro lado, el orden también permite observar las relaciones y nexos entre las diferentes cosas para ascender en el conocimiento hasta llegar a la verdad. En la Séptima regla, Descartes (1984) señala que "para completar la ciencia es preciso recorrer en un movimiento continuo e ininterrumpido del pensamiento todas y cada una de las cosas que conciernen a nuestro propósito, y abarcarlas en una enumeración suficiente y ordenada" (p. 95). Más adelante, escribe que el concepto de enumeración equivale a inducción y lo define como aquello de lo que se extrae una verdad (Descartes, 1984, p. 97). Igualmente, en la Novena regla define la enumeración como "una inferencia obtenida a partir de varias cosas separadas" (Descartes, 1984, p. 113).

Con todo, la Duodécima regla prescribe que es menester valerse de todos los recursos del entendimiento, de la imaginación, de los sentidos y de la memoria para ejecutar cada uno de los procesos de intuición, deducción y enumeración-comprobación. Hacerlo así asegura el acceso al conocimiento verdadero.

gen del saber (ciencia), tesis que plantearon mucho tiempo atrás Platón y Aristóteles. 


\section{UNA APROXIMACIÓN AL MÉTODO CARTESIANO. SU RELACIÓN CON LA CONTABILIDAD / C. VARGAS / 589}

\section{Influencia de la filosofía cartesiana en la contabilidad}

Es conveniente iniciar este acápite recordando que la idea de verdad es fundamental en la filosofía cartesiana. No obstante, la verdad en contabilidad resulta un asunto problemático no solo por los diferentes enfoques investigativos (ortodoxos y heterodoxos) con los cuales se construye teoría, que pueden implicar verdades diferentes; sino también por la construcción de variadas verdades en medio de la abundante regulación y la doctrina incorporadas en las prácticas contables. En este sentido, se podría afirmar que la contabilidad no posee o produce una única verdad sino múltiples verdades acordes con los intereses de los investigadores y agentes sociales. En las líneas siguientes se intenta describir la influencia de la idea de verdad y demás aportes planteados desde la filosofía cartesiana en los desarrollos contables.

Así, en los anales de la historia se le ha atribuido a Fray Luca Pacioli el haber dado origen a la teneduría de libros por el principio universal de la partida doble, gracias a un tratado especial sobre la contabilidad (Tractatus particularis de computis et scripturis) que incluyó en la que fuera considerada su más representativa obra, "Summa de arithmetica, geometria, proportioni et proportionalità", publicada hacia 1494, época renacentista. No obstante lo anterior, en muchos momentos se ha puesto en tela de juicio la originalidad de la obra de Pacioli. Se le acusa de haber plagiado el trabajo de Benedetto Cotrugli en lo que concierne a la creación de la partida doble. Sin embargo, es importante resaltar que Pacio- li fue el primer autor en publicar (de manera escrita) un tratado sobre la contabilidad.

En el tratado de Pacioli se explica el método de Venecia, un sistema de contabilidad utilizado en esta ciudad italiana que ya incorporaba los números arábigos y negativos, los cuales hicieron posible la aplicación del mencionado principio de la partida doble (De Sainte Croix, citado por Giménez-Barriocanal, 2003).

Ahora bien, en los siglos que siguieron a la época del Renacimiento inicia la modernidad (siglos XVI y XVII). Los aportes de Luca Pacioli son retomados por Fabio Besta y por el jesuita Ludovico Flori (Monagas, 2005). Este último escribió la obra Tratado de teneduría de libros, en la que presenta un estudio deductivo del balance general a partir de las cuentas del libro mayor (Ministerio de Hacienda de Costa Rica, 2011).

De acuerdo con la interpretación de Dulce Monagas (2005), Galileo Galilei "resalta la medición y la cuantificación por encima del método subjetivista, método que también se utilizó en el área contable" (p. 47) como se señalará posteriormente. Descartes, por su parte, rompe con el esquema filosófico de la época, al formular un nuevo método para acceder a la verdad (conocimiento) en el que la racionalidad humana se convierte en la mayor fuente del saber. A esto contribuyó su descubrimiento de la primera verdad: Cogito, ergo sum. Como se señaló anteriormente, en el método cartesiano cobran relevancia la razón y el pensamiento racional del hombre por encima del conocimiento sensible, por lo que las matemáticas constituyeron la ciencia por excelencia de Descartes y ello sustentado "por la certeza y evidencia que poseen 
sus razones" (Descartes, 2001, p. 43). De esta manera, confirmó los planteamientos de Galilei y consiguió que lo cuantificable y lo medible hicieran parte de la nueva filosofía y de la nueva forma de mirar (estudiar) el mundo, principalmente en el pensamiento de Occidente.

La contabilidad impone el modelo filosófico basado en la razón con su entrada a la universidad, lo que caracteriza su desarrollo racional que resultó legítimamente científico en el pensamiento moderno de Occidente (Gómez \& Ospina, 2009). Así, la racionalidad influenció algunos de los desarrollos teóricos e investigativos de la contabilidad entre los que se citan, a la luz de Wai Fong Chua (1986), los trabajos de:

- Pradip N. Khandwalla (1972), David C. Hayes (1977) y Vijay Govindarajan (1984) en torno a la teoría de la contingencia.

- Robert Libby (1975), Adrian M. Harrel (1977), Lawrence Kessler y Robert H. Ashton (1981) y Robert E. Hoskin (1983) sobre el aprendizaje por categorías probabilísticas.

- Ray Ball y Philip Brown (1968), Eugene F. Fama (1970), William H. Beaver y Roland E. Dukes (1973) y Nicholas J. Gonedes (1974) respecto de los mercados de capitales eficientes.

- Joel S. Demski y Gerald A. Feltham (1978), Jerold L. Zimmerman (1979) y Stanley Baiman (1982) sobre la teoría de la agencia y sistemas de control presupuestario.

Los trabajos mencionados han "constituido un intento por descubrir una realidad objetiva y conocible" (Chua, 1986, p. 44) y, por consiguiente, han consolidado lo que se ha denominado investigación positivista (tradición investigativa contable) enmarcada en la cuantificación, aplicación de modelos estocásticos y el criterio de objetividad e independencia entre sujeto y fenómeno de estudio, es decir, se otorga igual tratamiento a los fenómenos naturales y sociales. Desde este punto de vista, se puede señalar que el método y la racionalidad científica propuesta por Descartes contribuyeron al desarrollo de la contabilidad, como anotan Rosa Casal y Norka Viloria (2007).

Por su parte, Carlos Larrinaga (1999) plantea en la misma línea de pensamiento que la investigación predominante en contabilidad se ha orientado a la explicación de la naturaleza del orden social y por ello se estudian problemas que afectan a las organizaciones buscando mecanismos de control. En el mismo sentido, Wai Fong Chua (1986) afirma lo siguiente:

\footnotetext{
la corriente principal de la investigación contable está dominada por una creencia en el realismo físico — esto es, la concepción de que hay un mundo de realidad objetiva que existe de manera independiente a los seres humanos y que posee una naturaleza o esencia determinada que es susceptible de ser conocida- (p. 45).
}

Ahora bien, continuando con el hilo conductor de esta discusión, se retoman los planteamientos de Dulce Monagas (2005), quien identifica cierta influencia de la filosofía cartesiana sobre la contabilidad al señalar lo siguiente: 
El uso de la partida doble se somete a la razón por sobre todas las ideas, pues ya que esta no considera lo medible y cuantificable como válido, las transacciones como tales dejan de tener vigencia y no son entonces, objeto de estudio del conocimiento contable. Igualmente, desde el punto de vista deductivo surge el basamento teórico del Balance General, que se estudia como un conjunto de todas las cuentas del mayor [...]. La evidencia del arraigo de la filosofía cartesiana se manifiesta en la utilización del plano cartesiano como base para la representación gráfica de la transacción comercial del 'Libro Mayor' (p. 47).

Se puede colegir entonces que las posturas anteriores dan pie a la objetividad en la cuantificación y medición de los hechos (y fenómenos) por parte de la contabilidad, pensamiento propio del positivismo, el cual derivó en buena medida de la racionalidad científica de Descartes y que se ha consolidado como el paradigma ortodoxo en materia de teoría e investigación contable.

En este sentido, Rosa Casal y Norka Viloria (2002) exponen que la ciencia contable está impregnada de un paradigma cuantitativo, el cual es herencia de la filosofía cartesiana. Según este paradigma, todas las teorías debían transformarse en ecuaciones matemáticas para probar su cientificidad.

Desde esta perspectiva, puede afirmarse que lo medible y lo cuantificable se han impuesto en la mayoría de las áreas del conocimiento, lo cual consolidando de alguna manera el enfoque cuantitativo de investigación y el paradigma positivista de la ciencia. La contabilidad, desde la perspectiva de la representación financiera de los hechos, no ha estado ajena a esta tendencia y ha desarrollado una serie de principios generalmente aceptados en virtud de los cuales se pretende asegurar la representación fidedigna de los diferentes hechos económicos - criterio de verdad en la filosofía cartesiana-. Uno de esos principios contables es precisamente el de valuación y medición, a partir del cual han de valorarse las diferentes partidas de los estados financieros de manera confiable para garantizar en todo caso la revelación adecuada de las diferentes partidas. En el mismo sentido, los estándares internacionales de contabilidad contemplan el criterio de fiabilidad en la medición y el concepto de valor razonable para cumplir esta misma finalidad.

En esta línea, temas como la proporción y la matematización contempladas en el método cartesiano han sustentado algunos desarrollos de la contabilidad en materia de representación desde la perspectiva cuantitativa. A pesar de esto, no puede desconocerse la potencialidad de la contabilidad en el desarrollo del conocimiento y en la transformación de la realidad social desde sus perspectivas heterodoxas, como se expondrá más adelante.

Ahora bien, el método cartesiano también se puede ver aplicado en el proceso de confección de estados financieros, uno de los fines importantes que persigue la contabilidad en la medida en que proporciona información a "diferentes" usuarios para favorecer la toma de decisiones. De una parte, el precepto del análisis está dado en el conjunto de cuentas que componen el libro mayor, las cuales constitu- 
yen las partes más simples en que se reconocen contablemente los hechos. Posteriormente, en un proceso de síntesis (deducción) se obtienen el balance general, el estado de resultados y los demás informes financieros que revelan la realidad de manera sintética.

Por otra parte y continuando con la reflexión, se puede establecer una relación entre el método cartesiano y las prácticas contables. Este método está sustentado en la evidencia y en tal sentido, hay que dudar de todo a fin de asegurarse de que el conocimiento al que se accede es verdadero.

De acuerdo con Donaliza Cano, Danilo Lugo, José Eriberto Cano y Miguel Antonio Cano-Castaño (2009), la auditoría es el "examen objetivo, sistemático y profesional de las operaciones financieras o administrativas efectuado con posterioridad a su ejecución como servicio a la gerencia por personal de una unidad de auditoría, completamente independiente de dichas operaciones, con la finalidad de verificarlas, evaluarlas y elaborar un informe que contenga comentarios, conclusiones y recomendaciones, soportado en evidencias convincentes" (p. 314) (itálicas fuera del texto).

Pues bien, puede inferirse que la auditoría sustenta toda la ejecución del trabajo en la obtención de pruebas - evidencia-. A partir de esta se puede dictaminar o no la razonabilidad de las cifras contenidas en los estados financieros y se pueden hacer aseveraciones respecto al cumplimiento o no de determinadas disposiciones.

De igual manera, en la ejecución del trabajo de auditoría se aplica el segundo paso del método cartesiano, es decir, el análisis. En esta materia, el objeto de análisis debe ser descompuesto en tantas partes como sea necesario, las cuales son conocidas por medio de las técnicas de auditoría. Posteriormente, se reagrupan y se unen para establecer las relaciones entre ellas para detectar los hallazgos; es lo que corresponde a la etapa de la síntesis.

Por último, se hacen enumeraciones y revisiones de todo el trabajo de auditoría realizado. Precisamente, los denominados procedimientos de auditoría permiten consignar las etapas a desarrollar durante el análisis. De esta manera, el auditor se asegura de que no queda nada por fuera de su trabajo y se puede hacer una opinión (pensamiento racional) respecto de su trabajo, sustentada en la evidencia. Los papeles de trabajo permiten condensar los procedimientos aplicados y los análisis realizados. Los resultados obtenidos son finalmente plasmados en el informe de auditoría (dictamen en la auditoría financiera).

Es preciso aclarar que todo el trabajo de la auditoría debe estar enmarcado en la duda. Así, no puede darse una cifra como razonable hasta tener evidencia o prueba de que lo es, lo que implica que la duda se practique como principio en el campo de la auditoría y el control. De otro lado, se exige observar las cosas que estén justificadas y debidamente soportadas. En este sentido, se puede señalar que frente al hecho auditado se derivan evidencias que dan cuenta de la conformidad o no del hecho respecto de un estándar o procedimiento de auditoría, lo que posibilita la claridad frente al hecho. Cuando el auditor logra comprender la esencia del hecho y las situaciones que giran en torno a él, sus causas y sus con- 
secuencias, se puede hablar de la distinción en auditoría.

Así entonces, se puede establecer que la filosofía cartesiana impregna las prácticas contables, lo cual se hace visible en la auditoría como se mencionó anteriormente e incluso en otros subsistemas de la contabilidad. Solo por mencionar otro planteamiento que permita visualizar la correspondencia existente entre el método propuesto por Descartes y la praxis contable, se expone el proceso de elaboración de un diagnóstico financiero corporativo, entendido este como el análisis financiero que se hace sobre una organización, considerando variables cualitativas y cuantitativas, internas y del entorno. Óscar León García-Serna (1999) lo define como "el estudio que se hace de la información que proporciona la contabilidad y de toda la demás información disponible, para tratar de determinar la situación financiera de la empresa o de un sector específico de esta" (p. 190).

La necesidad de un diagnóstico en este sentido surge de la duda. Duda respecto de la situación de una organización: ¿Cómo estáen términos financieros-la empresa? Este interrogante solo se despeja en la medida en que se tenga evidencia sobre la posición de la empresa en materia de liquidez, endeudamiento, rentabilidad, eficiencia en la cobertura de costos y gastos, etc. Esta es precisamente la primera regla planteada por Descartes en el Discurso del método, es decir, la necesidad de evidencia. Como afirma Descartes (2001), no se debe "admitir como verdadera cosa alguna, como no supiese con evidencia que lo es" (p. 52).
Posteriormente, es menester descomponer el objeto de análisis en partes (segundo paso del método cartesiano). En el diagnóstico financiero se toman el balance general y el estado de resultados, y se construyen indicadores que permitan analizar cada una de las partes: liquidez; autonomía financiera, nivel de endeudamiento y cobertura de gastos financieros; lucratividad de la operación; eficiencia en la movilización de recursos y eficiencia en la cobertura de costos y gastos, entre otros. Igualmente, se analizan la composición de la inversión y el endeudamiento, el origen y la aplicación de recursos, las fuentes internas y externas de fondos, el comportamiento del mercado y del entorno en general, entre otros aspectos.

Después de esto se reagrupan las partes y se establecen relaciones entre la liquidez y la rentabilidad, entre esta y la movilización de recursos, entre la posición de equilibrio y la eficiencia en la cobertura de costos y gastos, etc. De igual manera, se determina la incidencia de las diferentes variables del entorno sobre la actividad empresarial. Adicionalmente, se deben hacer enumeraciones y revisiones a fin de asegurarse de que no queda nada por fuera del diagnóstico financiero. De esta manera, se da aplicación a las reglas III y IV del método cartesiano: síntesis y enumeraciones.

Por último, estos pasos del método (y del diagnóstico financiero) dan la posibilidad al analista y a los grupos de interés de acceder mediante el uso de la razón al conocimiento de la situación financiera de una organización. 


\section{La heterodoxia contable. Perspectivas alternativas a lo racional-objetivo}

Como se adelantó en líneas anteriores, desde la década de los setenta, es preciso advertir sobre la emergencia de otros paradigmas alternativos a la investigación predominante en contabilidad (interpretativo y sociocrítico) que, de manera antagónica, propenden primeramente por diferenciar los fenómenos sociales de los naturales y en tal sentido dan cabida a las subjetividades y se orientan hacia la construcción social del conocimiento sin hacer limitaciones dentro de lo meramente cuantificable o medible. Así, los fenómenos no se consideran acabados en términos absolutos, sino que el sujeto se aproxima a ellos con el propósito de interpretarlos y comprenderlos para finalmente contribuir a la transformación, en buen sentido, de la realidad social.

Así entonces, el criterio de verdad se debilita en contabilidad por cuanto esta (la contabilidad) no logra representar íntegra y totalmente la realidad. Luego, la objetividad y la racionalidad - legado de la filosofía cartesiana- resultan cuestionables en este sentido y de ahí la importancia de los nuevos enfoques (heterodoxia contable) para pensar e intervenir los diferentes hechos y fenómenos de la realidad.

En efecto, algunas de las presuposiciones de las perspectivas heterodoxas relacionadas por Anthony G. Puxty (citado en Larrinaga, 1999) son:

a. Los seres humanos actúan sobre las cosas —objetos, gente, organizaciones, ideas abstractas, actividades o situaciones-en función de los significados que tienen para ellos.

b. Los significados que las cosas tienen para los individuos son el resultado de la interacción social, es decir, las cosas no tienen un significado objetivo - anterior, independiente y externo al sujeto-.

c. Esos significados son manejados y modificados a través de un proceso interpretativo que se utiliza por cada individuo para tratar los signos que encuentra. Los significados no están ahí, sino que el individuo permanece activo en el proceso de interpretación (p. 114).

En el mismo sentido, Wai Fong Chua (1986) plantea en torno a la necesidad de la heterodoxia contable lo siguiente:

El pensamiento contable se modifica como los seres humanos y su entorno, y varía de la misma manera que lo hace la percepción de sus necesidades. Considerando este mutuo e interactivo acoplamiento entre el conocimiento y el mundo físico y humano, la producción de conocimiento está delimitada por las reglas hechas por el hombre y sus creencias, que definen el campo de acción del conocimiento, de los fenómenos empíricos, y la relación entre estos dos (p. 40).

Así, previo a terminar este escrito y con sustento en algunos argumentos planteados anteriormente, es importante tener en cuenta que no obstante lo expuesto es necesario que en contabilidad se incorporen otras visiones y enfoques como en efecto se ha hecho (hetero- 
doxia contable). Por ello, sin desconocer los interesantes aportes de la filosofía cartesiana, "en esta nueva sociedad, se pugna por instaurar una nueva razón contable con base en el nuevo paradigma de la ciencia, la complejidad dialógica, recursiva y hologramática de la realidad" (Casal \& Viloria, 2007, p. 24) que permita a la contabilidad interpretar diferentes fenómenos e intervenir la sociedad.

\section{Conclusiones}

El sustento teórico expuesto en este trabajo permite abstraer que la filosofía cartesiana es considerada el punto de partida de los pensamientos filosófico y científico moderno propiamente dichos por cuanto significó la imposición de la racionalidad en la ciencia y la derivación del positivismo, bajo el cual se han sumido muchos desarrollos en diferentes áreas de conocimiento, entre ellas, parte de los aportes efectuados desde la tradición investigativa de la contabilidad en materia de teoría e investigación contable.

Ahora bien, la influencia que ha tenido la filosofía cartesiana en la disciplina contable se ha presentado principalmente porque la lógica de la medición-cuantificación se ha impuesto en la corriente ortodoxa de la investigación contable (positivista). De igual manera, las prácticas contables han apropiado el modelo matemático para efectuar representaciones de la realidad, al considerar hechos y fenómenos que desde la lógica de la medición-cuantificación se puedan representar.

No obstante lo anterior, es de reconocer la existencia de paradigmas heterodoxos en la investigación contable, el interpretativo y el sociocrítico, bajo los cuales se establecen diferencias claras en la manera de concebir e intervenir los fenómenos sociales respecto de los naturales, lo que posibilita la existencia de subjetividades a partir de la integración sujeto-objeto y la construcción social del conocimiento.

En este sentido, se reconocen los aportes que desde la tradición investigativa se han efectuado en contabilidad puesto que estos han permitido, de una parte, avanzar en la consolidación del acervo del conocimiento científico y en el desarrollo de las prácticas contables. Por otra parte, el entendimiento y comprensión de esta tradición posibilitan explorar las perspectivas heterodoxas, al efectuar las pertinentes contrastaciones, mediante las cuales es posible intervenir los fenómenos de la realidad de manera diferente.

Así, no es conveniente rechazar de manera radical la ortodoxia o la heterodoxia contable. La complejidad de los diferentes fenómenos señalará la perspectiva que mejor permita aproximarse a los mismos, con lo cual se advierte sobre la necesidad de propiciar en la comunidad académica contable una coexistencia dialógica de las perspectivas a fin de fortalecer el estatus epistemológico de la contabilidad.

Por último, con este aporte se quiere animar el debate y la reflexión en el medio contable colombiano frente a los desarrollos que se han suscitado en la contabilidad, pero principalmente, respecto de las potencialidades que la disciplina posee para propiciar nuevos desarrollos con los cuales se pueda crear y re-crear las diferentes estructuras sociales. 


\section{Referencias}

Ávila-Fuenmayor, Francisco (2005). El dominio técnico: socavación del sujeto de vida. Opción, 21 (46), 124-140. Disponible en: http://www.redalyc.org/articulo. oa?id=31004606

Beuchot, Mauricio (2008). Perfiles esenciales de la hermenéutica. México: Fondo de Cultura Económica, FCE.

Cano, Donaliza; Lugo, Danilo; Cano, José Eriberto \& Cano-Castaño, Miguel Antonio (2009). Auditoría financiera forense en la investigación de delitos económicos $y$ financieros, lavado de dinero y activos, financiación del terrorismo. 3a ed. Bogotá: ECOE Ediciones.

Carvajal-Godoy, Johman (2007). El desarrollo del pensamiento moderno: la filosofía de la naturaleza de Descartes. Medellín: Universidad Pontifica Bolivariana.

Casal, Rosa A. \& Viloria, Norka (2002). La corriente positivista y su influencia en la ciencia contable. Actualidad Contable FACES, 005 (5), 7-22. Disponible en: http:// www.redalyc.org/pdf/257/25700505.pdf

Casal, Rosa A. \& Viloria, Norka (2007). La ciencia contable, su historia, filosofía, evolución y su producto. Actualidad Contable FACES, 10 (15), 19-28. Disponible en: http:// www.redalyc.org/pdf/257/25701503.pdf

Chua, Wai Fong (1986). Desarrollos radicales en el pensamiento contable. En Mauricio Gómez \& Carlos Mario Ospina (eds.). Avances interdisciplinarios para una comprensión crítica de la contabilidad. Textos paradigmáticos de las corrientes heterodoxas,
37-78. Medellín: Universidad de Antioquia y Universidad Nacional de Colombia.

Descartes, René (1984). Reglas para la dirección del espíritu. Madrid: Alianza Editorial.

Descartes, René (1997). Las pasiones del Alma. Madrid: Editorial Tecnos.

Descartes, René (2001). Discurso del método. Meditaciones metafísicas. Madrid: Editorial Libsa.

García-Serna, Óscar León (1999).

Administración financiera. Fundamentos y aplicaciones. $3^{\mathrm{a}}$ ed. Medellín: Óscar León García S.

Giménez-Barriocanal, Fernando (2003). La actividad económica en el derecho romano. Análisis contable. Madrid: Dykinson.

Gómez, Mauricio \& Ospina, Carlos Mario (2009). Ampliando las fronteras en la disciplina contable: una introducción para la contextualización de los ejemplares heterodoxos. En Mauricio Gómez \& Carlos Mario Ospina (eds.). Avances interdisciplinarios para una comprensión crítica de la contabilidad. Textos paradigmáticos de las corrientes heterodoxas, 13-35. Medellín: Universidad de Antioquia y Universidad Nacional de Colombia. Hernández-González, Francisco Javier \& Salgado-González, Sebastián (2010-2011). El racionalismo de Descartes. La preocupación por el método. Duererías, Cuadernos de Filosofía, 1-19. Disponible en: http://guindo. pntic.mec.es/ ssag0007/filosofica/Descartes. pdf

Hessen, Johannes (1997). Teoría del conocimiento. México: Losada Océano. 
UNA APROXIMACIÓN AL MÉTODO CARTESIANO. SU RELACIÓN CON LA CONTABILIDAD / C. VARGAS / 597

Hirschberger, Johannes (2000). Historia de la filosofía. Tomo II. $15^{\text {a }}$ ed. Barcelona: Herder. Larrinaga, Carlos (1999). Perspectivas alternativas de investigación en contabilidad: una revisión. Revista de Contabilidad, 3 (2), 103-131. Disponible en: http://www.rc-sar.es/verPdf. php?articleId $=45$

Luna-Alcoba, Manuel (2003). Descartes. Madrid: Editex.

Ministerio de Hacienda Costa Rica (2011). Breve reseña histórica de la Contabilidad Pública y de la Contabilidad Nacional del Ministerio de Hacienda. Disponible en: http://www.hacienda.go.cr/cifh/sidovih/ uploads/archivos/Articulo/Breve\%20 rese $\%$ C3\%B1a\%20hist\%C3\%B3rica\%20 de\%20la\%20Contabilidad\%20 P\%C3\%BAblica\%20y\%20de\%20la\%20 Contabilidad\%20Nacional\%20del\%20 Ministerio\%20de\%20Hacienda.pdf Monagas, Dulce (2005). El conocimiento contable. Actualidad Contable FACES, 08 (11), 45-51. Disponible en: http://www. redalyc.org/articulo.oa?id=25701106

Monroy-Nasr, Zuraya (2004). Razón y experiencia en el método cartesiano. Revista Digital Universitaria, 5 (3), 1-15. Disponible en: http://www.revista.unam.mx/vol.5/ num3/art13/art13.htm, http://www.revista. unam.mx/vol.5/num3/art13/mar_art13.pdf
Pacioli, Luca (1962). Summa de arithmetica, geometria, proportioni et proportionalità. México: Instituto Mexicano de Contadores Públicos, IMCP.

Perilla-Amórtegui, Édgar \& Valderrama-Prieto, Marcos (1999). Realidad económica contable en Colombia. Bogotá: Centro Editorial Javeriano, CEJA.

Quintás-Alonso, Guillermo; Gracia-Calandín, Javier; Larraz, Diego; Moreno, E; Palanca, P \& Ruiz, E. (2009). Descartes. Leyendo el discurso del método. Valencia, España: Publicaciones de la Universitat de València.

Ryan, Bob; Scapens, Robert W. \& Theobald, Michael (2004). Metodología de la investigación en finanzas y contabilidad. Carlos Ganzinelli (trad.). Barcelona: Deusto.

- Fecha de recepción: 23 de julio de 2013

- Fecha de aceptación: 28 de enero de 2014

- Disponible en línea: 01 de julio de 2014

\section{Para citar este artículo}

Vargas-Restrepo, Carlos Mario (2014). Una aproximación al método cartesiano. Su relación con la contabilidad. Cuadernos de Contabilidad, 15 (38), 575-597.

doi: 10.11144/Javeriana.cc15-38.uamc 
